\title{
Far-infrared properties of submillimeter and optically faint radio galaxies ${ }^{\star}$
}

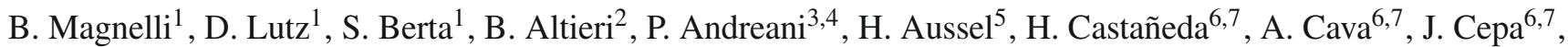
A. Cimatti ${ }^{8}$, E. Daddi ${ }^{5}$, H. Dannerbauer ${ }^{5}$, H. Dominguez ${ }^{9}$, D. Elbaz ${ }^{5}$, N. Förster Schreiber ${ }^{1}$, R. Genzel ${ }^{1}$, A. Grazian ${ }^{10}$, C. Gruppioni ${ }^{9}$, G. Magdis ${ }^{5}$, R. Maiolino ${ }^{10}$, R. Nordon ${ }^{1}$, I. Pérez Fournon ${ }^{6,7}$, I. Pérez García ${ }^{2}$, A. Poglitsch ${ }^{1}$, P. Popesso ${ }^{1}$, F. Pozzi ${ }^{8}$, L. Riguccini ${ }^{5}$, G. Rodighiero ${ }^{11}$, A. Saintonge ${ }^{1}$, P. Santini ${ }^{10}$, M. Sanchez-Portal ${ }^{2}$, L. Shao ${ }^{1}$, E. Sturm ${ }^{1}$, L. Tacconi ${ }^{1}$, I. Valtchanov ${ }^{5}$, E. Wieprecht ${ }^{1}$, and E. Wiezorrek ${ }^{1}$

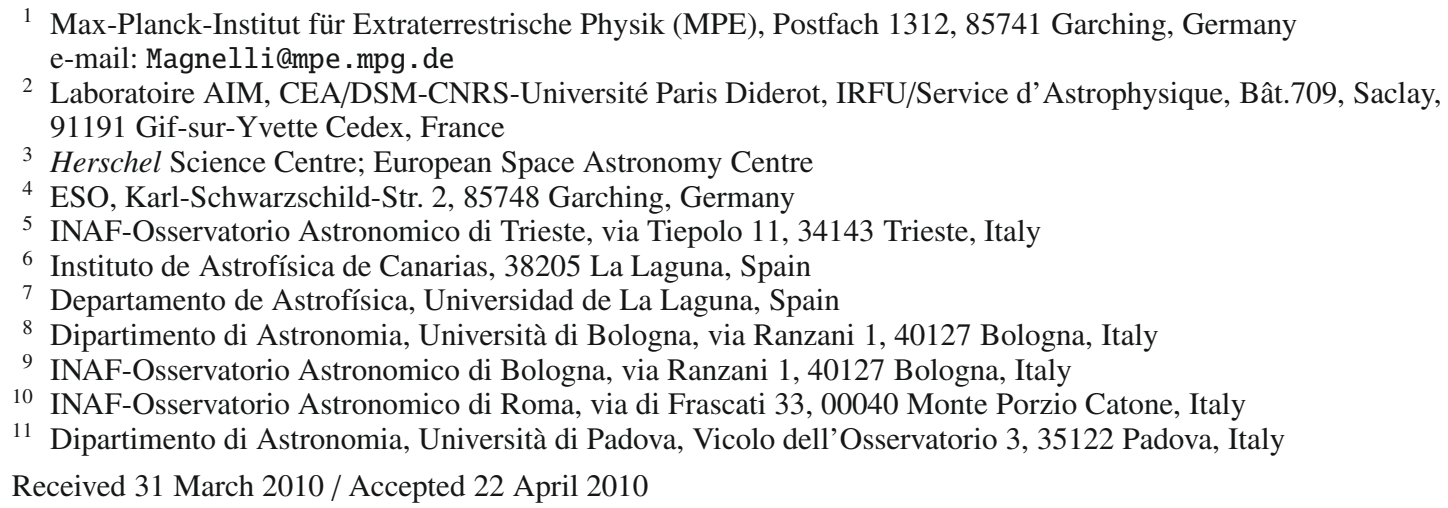

\section{ABSTRACT}

\begin{abstract}
We use deep observations obtained with the Photodetector Array Camera and Spectrometer (PACS) onboard the Herschel Space Observatory to study the far-infrared (FIR) properties of submillimeter and optically faint radio galaxies (SMGs and OFRGs). From literature we compiled a sample of 35 securely identified SMGs and nine OFRGs located in the GOODS-N and the A2218 fields. This sample is cross-matched with our PACS $100 \mu \mathrm{m}$ and $160 \mu \mathrm{m}$ multi-wavelength catalogs based on sources-extraction using prior detections at $24 \mu \mathrm{m}$. About half of the galaxies in our sample are detected in at least the PACS $160 \mu \mathrm{m}$ bandpass. The dust temperatures and the infrared luminosities of our galaxies are derived by fitting their PACS and SCUBA $850 \mu \mathrm{m}$ (only the upper limits for the OFRGs) flux densities with a single modified $(\beta=1.5)$ black body function. The median dust temperature of our SMG sample is $T_{\text {dust }}=36 \pm 8 \mathrm{~K}$ while for our OFRG sample it is $T_{\text {dust }}=47 \pm 3 \mathrm{~K}$. For both samples, median dust temperatures derived from Herschel data agree well with previous estimates. In particular, Chapman et al. $(2005, \mathrm{ApJ}, 622,772)$ found a dust temperature of $T_{\text {dust }}=36 \pm 7 \mathrm{~K}$ for a large sample of SMGs assuming the validity of the FIR/radio correlation (i.e., $q=\log _{10}\left(L_{\mathrm{FIR}}[\mathrm{W}] / L_{1.4 \mathrm{GHz}}\left[\mathrm{W} \mathrm{Hz}{ }^{-1}\right] / 3.75 \times 10^{12}\right)$ ). The agreement between our studies confirms that the local FIR/radio correlation effectively holds at high redshift even though we find $\langle q\rangle=2.17 \pm 0.19$, a slightly lower value than that observed in local systems. The median infrared luminosities of SMGs and OFRGs are $4.6 \times 10^{12} L_{\odot}$ and $2.6 \times 10^{12} L_{\odot}$, respectively. We note that for both samples the infrared luminosity estimates from the radio part of the spectral energy distribution (SED) are accurate, while estimates from the mid-IR are considerably $(\sim \times 3)$ more uncertain. Our observations confirm the remarkably high luminosities of SMGs and thus imply median star-formation rates of $960 M_{\odot} \mathrm{yr}^{-1}$ for SMGs with $S(850 \mu \mathrm{m})>5 \mathrm{mJy}$ and $460 M_{\odot} \mathrm{yr}^{-1}$ for SMGs with $S(850 \mu \mathrm{m})>2 \mathrm{mJy}$, assuming a Chabrier IMF and no dominant AGN contribution to the far-infrared luminosity.
\end{abstract}

Key words. infrared: galaxies - submillimeter: galaxies - galaxies: evolution - galaxies: starburst

\section{Introduction}

Herschel observations probe the rest-frame far-infrared emission of high-redshift galaxies. Thus, they provide for the first time robust estimates of the infrared luminosities of these high-redshift galaxies and test previous measurements that were based on extrapolation from shorter or longer wavelengths. We here focus on

* Herschel is an ESA space observatory with science instruments provided by European-led Principal Investigator consortia and with important participation from NASA. two populations of high-redshift star-forming galaxies selected at submillimeter (submm) and radio wavelengths.

Since their discovery in the late 1990s, submillimeter galaxies (SMGs) have become the selection of choice for the most luminous tail of the high-redshift star-forming galaxy population. It has been found that SMGs have a typical redshift of $z \sim 2$ (Chapman et al. 2005; Pope et al. 2006), are massive systems $\left(M_{\star} \sim 10^{10}-10^{11} M_{\odot}\right.$, Swinbank et al. 2004; Tacconi et al. 2006) and are compact (e.g., Tacconi et al. 2008). 
Interferometric observations of their $\mathrm{CO}$ molecular gas suggest that the most luminous SMGs $\left(S_{850 \mu \mathrm{m}}>5 \mathrm{mJy}\right)$ are merging sytems (Tacconi et al. 2006, 2008) with high star-formation efficiencies compared to typical galaxies of a similar mass (Daddi et al. 2008). Therefore, these SMGs are thought to exhibit very intense $\left(S F R \sim 1000 M_{\odot} \mathrm{yr}^{-1}\right)$ short-lived star-formation bursts triggered by mergers and to be the high-redshift progenitors of local massive early-type galaxies (Daddi et al. 2007a,b; Tacconi et al. 2008; Cimatti et al. 2008).

Although SMGs provide a powerful tool to constrain the formation and the evolution of high-redshift dusty star-forming galaxies, their selection is subject to strong biases. In particular, because submm observations probe the blackbody emission of dust in the Rayleigh-Jeans regime, they are strongly anti-correlated with the dust temperature $\left(S_{850} \propto T_{\text {dust }}^{-3.5}\right)$. For a given infrared luminosity, galaxies with hot dust might fall below the detection limit of current submm instruments. The first observational evidence of a missing population of highredshift dusty star-forming galaxies with hot dust has been given by Chapman et al. (2004) using a selection of radio-detected but submm-faint galaxies with UV spectra consistent with highredshift starbursts. These optically faint-radio galaxies (OFRGs) have a comoving volume density (i.e., $\sim 10^{-5} \mathrm{Mpc}^{-3}$ at $1<z<3$, Chapman et al. 2004), stellar masses and sizes comparable to SMGs, and some have a dust temperature of $\sim 52 \mathrm{~K}$ (Casey et al. 2009a,b).

While SMGs and OFRGs are an important component of the high-redshift massive galaxy population, many of their fundamental properties still rely on indirect measurements. In particular, direct determinations of SMG dust temperatures were limited (Kovács et al. 2006) because they were not done using rest-frame far-infrared observations on both sides of the peak of the SEDs. More importantly, their infrared luminosites are still debated. Indeed, because theoretical simulations of galaxy evolution have had great difficulties to account for the current inferred luminosities/star-formation rates and number counts (Baugh et al. 2005; Davé et al. 2010), they still question whether these luminosities have been overestimated or whether the IMF is significantly more top-heavy than in the local Universe.

Using deep observations by the Photodetector Array Camera and Spectrometer (PACS; Poglitsch et al. 2010) onboard the Herschel Space Observatory (Pilbratt et al. 2010) we will have for the first time robust estimates of the dust temperatures and the infrared luminosities of SMGs and OFRGs. Throughout the paper we use a cosmology with $H_{0}=70 \mathrm{~km} \mathrm{~s}^{-1} \mathrm{Mpc}^{-1}, \Omega_{\Lambda}=0.7$ and $\Omega_{\mathrm{M}}=0.3$.

\section{Observations}

In this study we used deep PACS $100 \mu \mathrm{m}$ and $160 \mu \mathrm{m}$ observations of the Great Observatories Origins Deep SurveyNorth (GOODS-N; $12^{\mathrm{h}} 36^{\mathrm{m}},+62^{\circ} 14^{\prime}$ ) and the Abell 2218 $\left(16^{\mathrm{h}} 35^{\mathrm{m}},+66^{\circ} 12^{\prime}\right)$ fields. These observations were taken as part of the PACS evolutionary probe $\left(\mathrm{PEP}^{1}\right)$ guaranteed time key program. The GOODS-N field covers a region of $10^{\prime} \times 15^{\prime}$ $(30 \mathrm{~h})$, while the deep part of the A2218 field covers a region of $4^{\prime} \times 4^{\prime}(13 \mathrm{~h})$.

At the resolution of Herschel, all sources in our fields are point sources (i.e. FWHM $\sim 8^{\prime \prime}\left[12^{\prime \prime}\right]$ at $100 \mu \mathrm{m}[160 \mu \mathrm{m}]$ ). Flux densities are hence estimated using a point spread functionfitting technique based on prior source positions detected at $24 \mu \mathrm{m}$. The use of priors provides a straightforward association

${ }^{1}$ http://www.mpe.mpg.de/ir/Research/PEP between the IRAC, MIPS and PACS sources. Using Monte Carlo simulations we estimate the quality of our PACS $100 \mu \mathrm{m}$ and $160 \mu \mathrm{m}$ catalogs, i.e. photometric error, completeness and contamination as a function of the flux density. In the GOODS-N field our observations reach a $3 \sigma$ limit of $\sim 3 \mathrm{mJy}$ and $\sim 5.7 \mathrm{mJy}$ at $100 \mu \mathrm{m}$ and $160 \mu \mathrm{m}$ respectively, while in the A2218 field they reach a $3 \sigma$ limit of $\sim 2.5 \mathrm{mJy}$ and $\sim 5 \mathrm{mJy}$ at $100 \mu \mathrm{m}$ and $160 \mu \mathrm{m}$ respectively.

A complete description of PEP data reduction and sources extraction is given in Appendix A of Berta et al. (2010).

\section{Galaxy sample}

To obtain a robust measurement of the dust temperature and infrared luminosity of a given galaxy one needs to have an accurate estimate of its redshift. Consequently, we decided to restrict our study to a sample of SMGs and OFRGs with accurate redshift estimates derived from secured radio/mid-infrared identifications (PACS identifications of SMGs are presented in Dannerbauer et al. in prep). In the GOODS-N field, our SMG sample is based on multi-wavelength identifications of SCUBA and AzTEC sources made by Pope et al. (2006) $)^{2}$ and Chapin et al. (2009), respectively. SMGs with tentative redshifts determined from their IRAC or mid/far-infrared/radio colors were excluded from our sample. Sources with multiple optical counterparts (GN04, GN07, GN19 and GN39) were treated as a single system (i.e. we will use the sum of the radio and midinfrared flux from the two components when determining their far-infrared properties) because they are all thought to be interacting galaxies (Pope et al. 2006). All these different criteria yield an SMG sample containing 29 sources in the GOODS-N field. In the A2218 field, our SMG sample is assembled from the literature (Kneib et al. 2004; Knudsen et al. 2006, 2008) and contains six lensed sources. Because these galaxies are magnified, their mid-to-far infrared fluxes were de-magnified prior to further analysis using magnification factors from the above references. Among these six lensed sources, three correspond to the same lensed galaxy (SMMJ16359+6612; Kneib et al. 2004). Finally, our OFRG sample is taken from Casey et al. (2009a,b) and contains nine sources, all situated in the GOODS-N field. We note that all but three sources of our entire sample (i.e. SMGs and OFRGs) have spectroscopic redshifts.

The SMG and OFRG samples were cross-matched with our PACS multi-wavelength catalogs using a matching radius of $3^{\prime \prime}$. We detected 19 out of 35 SMGs in at least the PACS $160 \mu \mathrm{m}$ bandpass (17 out of 33 if not multi-counting the 3-component lensed source detected in A2218). The PACS sample is slightly biased towards lower redshift sources because of the positive $K$-correction: while the median redshift of our parent SMG sample is $z=2$, the median redshift of our PACS detected SMG sample is $z=1.7$. Five out of nine OFRGs have PACS $100 \mu \mathrm{m}$ and $160 \mu \mathrm{m}$ detections. This sample is also slightly biased toward lower redshift $(z=1.5)$ because the OFRG situated at the highest redshift is undetected in our PACS images.

We note that our SMG sample contains sources with $2 \mathrm{mJy}<$ $S_{850 \mu \mathrm{m}}<5 \mathrm{mJy}$, while the most luminous tail of the SMG population, mostly associated with major mergers, is defined using $S_{850 \mu \mathrm{m}}>5 \mathrm{mJy}$. Below, we will draw our conclusions distinguishing these two populations of SMGs.

\footnotetext{
2 For GN05, GN07, GN10, GN20 and GN20.2, we used the spectroscopic redshifts revised in Pope et al. (2008) and Daddi et al. (2009a,b).
} 


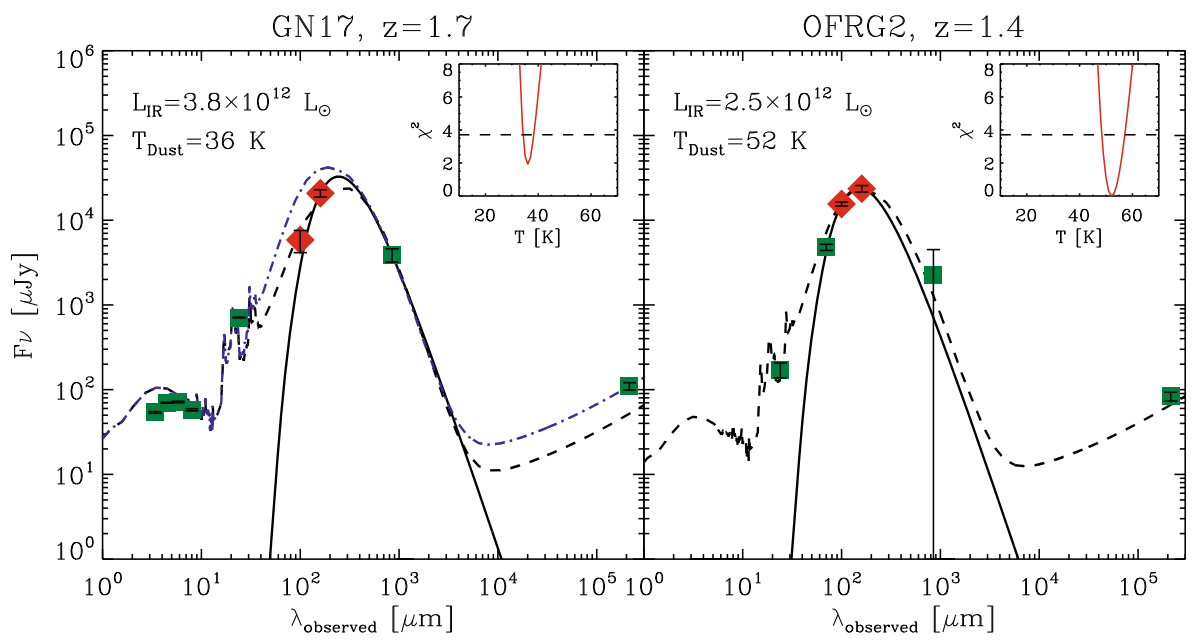

Fig. 1. Spectral energy distribution (SED) of one SMG (left) and one OFRG (right). Red diamonds present our PACS measurements while green squares present the multi-wavelength ancillary data taken from the literature (Pope et al. 2006; Casey et al. 2009a,b). The modified blackbody emission $(\beta=1.5)$ best-fitting the data are shown by solid lines. Dashed lines present the Dale \& Helou SED template bestfitting the mid- to far-infrared observations. In the left panel, the blue dotted-dashed line shows the Dale \& Helou SED template bestfitting the submm and radio photometries. The inset in each panel shows $\chi^{2}$ vs. $T_{\text {dust }}$.

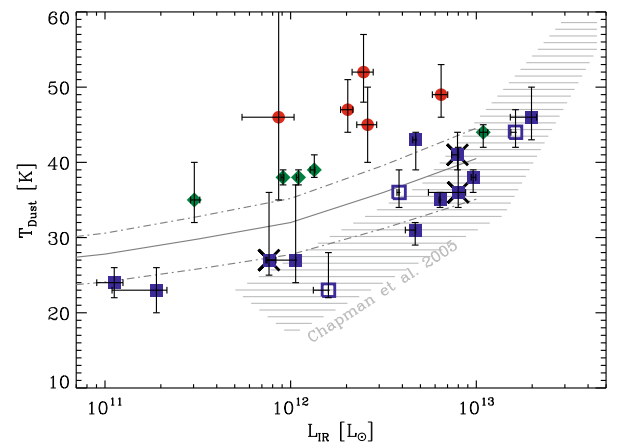

Fig. 2. Dust temperature-luminosity relation. The filled blue squares and opened blue squares denote SMGs located in the GOODS-N fields with spectroscopic and photometric redshift, respectively. The crosses denote sources which contain an AGN, as indicated by the presence of hard X-rays. The filled green diamonds present SMGs located in the A2218 field (the three data points with $L_{\mathrm{IR}} \sim 1 \times 10^{12} L_{\odot}$ correspond to the same lensed galaxy). The filled red circles are from our OFRGs sample. The striped area presents results for SMGs extrapolated by Chapman et al. (2005) from radio and submm data. The Chapman et al. (2003) derivation of the median and interquartile range of the $T_{\text {dust }}-L_{\mathrm{IR}}$ relation observed at $z \sim 0$ is shown by solid and dasheddotted lines, linearly extrapolated to $10^{13} L_{\odot}$.

\section{Data analysis}

In order to infer the dust temperature of our galaxies we fitted their PACS and SCUBA photometry (only the upper limit for the OFRGs) with a modified blackbody function, with a dust emissivity $\beta=1.5$ (see Fig. 1). Their total infrared luminosities $\left(L_{\mathrm{IR}}[8-1000 \mu \mathrm{m}]\right)$ were inferred from these best fits using the far-infrared luminosity definition $\left(L_{\mathrm{FIR}}[40-120 \mu \mathrm{m}]\right)$ given by Helou et al. (1988) and a color-correction term (Dale et al. $\left.2001, L_{\mathrm{IR}}=1.91 \times L_{\mathrm{FIR}}\right)$.

We adopted a single dust temperature characterization because studies of IRAS galaxies have demonstrated that this provides an accurate diagnostic of the typical heating condition in their interstellar medium (Desert et al. 1990). While for most of our galaxies this single dust temperature characterization provides a good description of their far-infrared SED, for 6 SMGs, this single dust temperature model yields high $\chi^{2}$ values (i.e. $\left.\chi^{2}>2.71+N_{\text {dof }}\right)$. All these galaxies appear either to be the more distant ones or to exhibit far-infrared colors typical of very cold systems. In both cases, their PACS $100 \mu \mathrm{m}$ flux densities might by contaminated by a hotter dust component. Therefore we excluded their PACS $100 \mu \mathrm{m}$ photometry from the fit and recomputed their dust temperatures. We note that excluding the

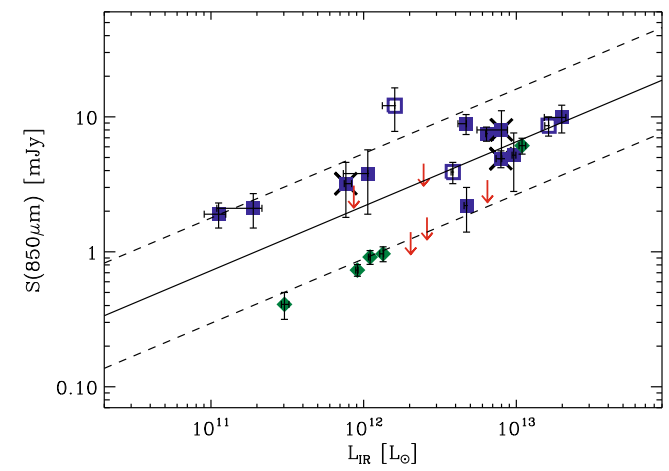

Fig. 3. Submm flux densities as function of the infrared luminosity. The symbols are same as in Fig. 2 except for the OFRGs, for which we have only upper limits. The solid and dashed lines show the linear fit to the $S_{850 \mu \mathrm{m}}-L_{\mathrm{IR}}$ relation and the $1 \sigma$ envelop $\left(L_{\mathrm{IR}}\left[L_{\odot}\right]=10^{11.3 \pm 0.8} \times\right.$ $\left.S_{850 \mu \mathrm{m}}^{2.1 \pm 0.1}[\mathrm{mJy}]\right)$. If we remove the lensed-SMGs of A2218 from the fit, we find a weaker correlation $\left(L_{\mathrm{IR}}\left[L_{\odot}\right]=10^{10.0 \pm 0.4} \times S_{850 \mu \mathrm{m}}^{3.5 \pm 0.1}[\mathrm{mJy}]\right)$.

PACS $100 \mu \mathrm{m}$ photometry from the fit of all our galaxies changes their median dust temperature by only $\Delta T_{\text {dust }} \sim-2 \mathrm{~K}$.

We tested that our results are relatively insensitive to $\beta$ and to our single dust component characterization. Indeed we note that using $\beta=2$, we found only small differences in the values of $T_{\text {dust }}\left(\Delta T_{\text {dust }} \sim+3 \mathrm{~K}\right)$. Moreover, we also note that to fit the PACS and SCUBA photometry with the multiple dust components model of Dale \& Helou (2002) yields rest-frame infrared colors $\left(S_{60 \mu \mathrm{m}} / S_{100 \mu \mathrm{m}}\right)$, or equivalently $T_{\text {dust }}$, which excellently agree with those inferred from our blackbody analysis.

\section{Discussion}

Figure 2 depicts the locations of our SMGs and OFRGs on the $T_{\text {dust }}-L_{\mathrm{IR}}$ plane and Fig. 3 shows their locations on the $S_{850 \mu \mathrm{m}}-L_{\mathrm{IR}}$ plane. As already mentioned, OFRGs are biased towards hot dust temperatures; their median $T_{\text {dust }}$ is of $47 \pm 3 \mathrm{~K}$ and their median $L_{\mathrm{IR}}$ is $2.6 \times 10^{12} L_{\odot}$. In contrast, SMGs have lower dust temperatures with median $T_{\text {dust }}=36 \pm 8 \mathrm{~K}$ and $L_{\mathrm{IR}}=4.6 \times 10^{12} L_{\odot}$. We note that lensed-SMGs from A2218 and with $L_{\mathrm{IR}}<2 \times 10^{12} L_{\odot}$ exhibit intermediate dust properties and are less biased towards cold dust temperatures than the entire SMG sample. This is because these galaxies would have escaped both the SMG and OFRG selection method without magnification. We also note that bright SMGs (i.e. $S_{850 \mu \mathrm{m}}>5 \mathrm{mJy}$ ) have higher median infrared luminosities $\left(L_{\mathrm{IR}}=9.6 \times 10^{12} L_{\odot}\right)$ and higher median dust temperatures $\left(T_{\text {dust }}=38 \mathrm{~K}\right)$ than the entire 
SMG sample because there is a correlation between $S_{850 \mu \mathrm{m}}$ and $L_{\mathrm{IR}}$ (Fig. 3). These estimates are the first direct observational measurements of the dust temperatures and the infrared luminosities of SMGs and OFRGs.

Our observations reveal that high redshift dusty star-forming galaxies exhibit a wide range of dust temperatures. In particular at low infrared luminosities $\left(L_{\mathrm{IR}}<4 \times 10^{12} L_{\odot}\right)$ the dust temperature dispersion observed in our sample might suggest a higher $T_{\text {dust }}-L_{\mathrm{IR}}$ scatter than that observed by Chapman et al. (2003) at $z \sim 0$. Nevertheless, this conclusion is most likely driven by selection effects because a significant fraction of the galaxies with intermediate dust properties were probably missed by our current sample. Indeed, we note that studying a $L_{\mathrm{IR}}$-selected sample of galaxies observed with Herschel, Hwang et al. (in prep) find modest changes in the $T_{\text {dust }}-L_{\mathrm{IR}}$ relation as function of the redshift: at $z>0.5$, galaxies with $L_{\mathrm{IR}}>5 \times 10^{10} L_{\odot}$ are slightly colder $(\sim 3 \mathrm{~K})$ than local ones and the scatter of the $T_{\text {dust }}-L_{\mathrm{IR}}$ relation slightly increase at high redshift.

Though previous estimates of the dust temperatures of SMGs and OFRGs relied on indirect observations, they agree relatively well with our measurements. In particular, Chapman et al. (2005) found a dust temperature of $T_{\text {dust }}=36 \pm 7 \mathrm{~K}$ for a large sample of SMGs assuming the validity of the FIR/radio correlation. In order to establish this agreement on a common sample, we applied the same method as Chapman et al. (2005) to our SMG sample, i.e. we fitted the radio and $850 \mu$ m photometries with dust SED templates from Dale \& Helou (2002) and then translated them into $T_{\text {dust }}$ using their $R(60,100)$ to $T_{\text {dust }}$ map. With this method we found higher $T_{\text {dust }}$ (by $\sim 4 \mathrm{~K}$ ) and $L_{\mathrm{IR}}$ $(\sim \times 1.5$ times) than what we obtained using our blackbody analysis. These discrepancies arise because the Dale \& Helou SED templates assume a FIR/radio correlation with $\langle q\rangle=2.34$ (Yun et al. 2001), while in our samples we find $\langle q\rangle=2.17 \pm 0.19$ (see also Ivison et al. 2010b). Although this value of $\langle q\rangle$ is still in line with results from local systems (which have a dispersion of $0.19 \mathrm{dex}$ ), it is also consistent with an evolution of $\langle q\rangle$ proportional to $(1+z)^{-0.15 \pm 0.03}$ as found by Ivison et al. (2010a).

Our results reveal that one can obtain a very reliable estimate of the infrared luminosity of a given galaxy from its radio flux density $\left(\sigma\left[\log _{10}\left(L_{\mathrm{IR}}^{\text {Radio }} / L_{\mathrm{IR}}^{\text {Blackboby }}\right)\right] \sim 0.18 \mathrm{dex}\right)$ using $\langle q\rangle=2.17$. In contrast, the use of the $24 \mu \mathrm{m}$ emission and of the Chary \& Elbaz SED library yields an inaccurate estimate of the infrared luminosity characterized by a large scatter $\left(\sigma\left[\log _{10}\left(L_{\mathrm{IR}}^{24 \mu \mathrm{m}} / L_{\mathrm{IR}}^{\text {blackbody }}\right)\right] \sim 0.48 \mathrm{dex}\right)$ and a systematic overestimation $(\sim \times 2$ times) of the most luminous galaxies $\left(L_{\mathrm{IR}}>4 \times 10^{12} L_{\odot}\right)$. We thus find that for the very luminous infrared galaxies studied here, luminosity extrapolations based on the radio emission are considerably more reliable than those based on the mid-infrared emission (see also Elbaz et al. 2010; and Nordon et al. 2010).

Using $\langle q\rangle=2.17$, we can predict the infrared luminosities of our PACS undetected SMGs. Then, using the Dale \& Helou SED templates normalized to these infrared luminosities, we can fit their $850 \mu \mathrm{m}$ photometries. We find that for 13 out of 16 undetected SMGs, PACS flux densities inferred using these fits are below the detection threshold of our observations. Of the three sources with PACS fluxes predictions above our detection threshold, one is known to be contaminated by an AGN and the other two are suspected to have wrong redshift estimates (Daddi et al. 2009b). The PACS nondetections are thus fully consistent with the properties inferred from the detections. This analysis cannot be performed on our PACS undetected OFRGs because they are also undetected at the SCUBA wavelength.
Our observations unambiguously confirm the remarkably large infrared luminosities of bright SMGs (i.e. $S_{850 \mu \mathrm{m}}>5 \mathrm{mJy}$ ) which correspond to SFRs of $960 M_{\odot} \mathrm{yr}^{-1}\left(S F R\left[M_{\odot} \mathrm{yr}^{-1}\right]=\right.$ $1 \times 10^{-10} L_{\mathrm{IR}}\left[L_{\odot}\right]$, assuming a Chabrier IMF and no dominant AGN contribution to the far-infrared luminosity). Such high SFRs are difficult to reconcile with secular evolution (e.g. Davé et al. 2010) and could correspond to a merger-driven stage in the evolution of these galaxies. This hypothesis is supported by $\mathrm{CO}$ observations of bright SMGs which have revealed large CO linewidths and disturbed gas morphologies (Tacconi et al. 2008). Our observations also confirm that OFRGs exhibit higher dust temperatures than faint SMGs $\left(S_{850 \mu \mathrm{m}}<5 \mathrm{mJy}\right)$ observed at the same redshift and with equivalent infrared luminosities. While the relatively low median SFRs of OFRGs and faint SMGs (260 $M_{\odot} \mathrm{yr}^{-1}$ and $106 M_{\odot} \mathrm{yr}^{-1}$ respectively) could be explained by secular evolution, we need to understand why they exhibit different dust temperatures and to study a possible link with bright SMGs. A clear evolutionary picture will require detailed studies of the dust and molecular gas distribution in a sample of highredshift star-forming galaxies unbiased towards any particular dust temperature. This SFR-selected sample can now be built with our ongoing deep Herschel observations.

Acknowledgements. PACS has been developed by a consortium of institutes led by MPE (Germany) and including UVIE (Austria); KU Leuven, CSL, IMEC (Belgium); CEA, LAM (France); MPIA (Germany); INAFIFSI/OAA/OAP/OAT, LENS, SISSA (Italy); IAC (Spain). This development has been supported by the funding agencies BMVIT (Austria), ESA-PRODEX (Belgium), CEA/CNES (France), DLR (Germany), ASI/INAF (Italy), and CICYT/MCYT (Spain).

\section{References}

Baugh, C. M., Lacey, C. G., Frenk, C. S., et al. 2005, MNRAS, 356, 1191 Berta, S., et al. 2010, A\&A, 518, L30

Casey, C. M., Chapman, S. C., Beswick, R. J., et al. 2009a, MNRAS, 399, 121 Casey, C. M., Chapman, S. C., Neri, R., et al. 2009b, MNRAS, submitted [arXiv: 0910. 5756]

Chapin, E. L., Pope, A., Scott, D., et al. 2009, MNRAS, 398, 1793

Chapman, S. C., Helou, G., Lewis, G. F., \& Dale, D. A. 2003, ApJ, 588, 186 Chapman, S. C., Smail, I., Blain, A. W., \& Ivison, R. J. 2004, ApJ, 614, 671 Chapman, S. C., Blain, A. W., Smail, I., \& Ivison, R. J. 2005, ApJ, 622, 772 Cimatti, A., Cassata, P., Pozzetti, L., et al. 2008, A\&A, 482, 21

Daddi, E., Alexander, D. M., Dickinson, M., et al. 2007a, ApJ, 670, 173

Daddi, E., Dickinson, M., Morrison, G., et al. 2007b, ApJ, 670, 156

Daddi, E., Dannerbauer, H., Elbaz, D., et al. 2008, ApJ, 673, L21

Daddi, E., Dannerbauer, H., Krips, M., et al. 2009a, ApJ, 695, L176

Daddi, E., Dannerbauer, H., Stern, D., et al. 2009b, ApJ, 694, 1517

Dale, D. A., \& Helou, G. 2002, ApJ, 576, 159

Dale, D. A., Helou, G., Contursi, A., Silbermann, N. A., \& Kolhatkar, S. 2001, ApJ, 549, 215

Davé, R., Finlator, K., Oppenheimer, B. D., et al. 2010, MNRAS, 404, 1355

Desert, F.-X., Boulanger, F., \& Puget, J. L. 1990, A\&A, 237, 215

Elbaz, D., et al. 2010, A\&A, 518, L29

Helou, G., Khan, I. R., Malek, L., \& Boehmer, L. 1988, ApJS, 68, 151

Ivison, R. J., Alexander, D. M., Biggs, A. D., et al. 2010a, MNRAS, 402, 245

Ivison, R. J., et al. 2010b, A\&A, 518, L31

Kneib, J., van der Werf, P. P., Kraiberg Knudsen, K., et al. 2004, MNRAS, 349, 1211

Knudsen, K. K., Barnard, V. E., van der Werf, P. P., et al. 2006, MNRAS, 368, 487

Knudsen, K. K., van der Werf, P. P., \& Kneib, J. 2008, MNRAS, 384, 1611

Kovács, A., Chapman, S. C., Dowell, C. D., et al. 2006, ApJ, 650, 592

Nordon, R., et al. 2010, A\&A, 518, L24

Pilbratt, G. L., et al. 2010, A\&A, 518, L1

Poglitsch, A., et al. 2010, A\&A, 518, L2

Pope, A., Chary, R., Alexander, D. M., et al. 2008, ApJ, 675, 1171

Pope, A., Scott, D., Dickinson, M., et al. 2006, MNRAS, 370, 1185

Swinbank, A. M., Smail, I., Chapman, S. C., et al. 2004, ApJ, 617, 64

Tacconi, L. J., Neri, R., Chapman, S. C., et al. 2006, ApJ, 640, 228

Tacconi, L. J., Genzel, R., Smail, I., et al. 2008, ApJ, 680, 246

Yun, M. S., Reddy, N. A., \& Condon, J. J. 2001, ApJ, 554, 803 